\title{
Análisis del COVID-19 y probabilidades de fallecimiento para los municipios de los alrededores de Bogotá
}

\section{Analysis of COVID and probabilities of death for the municipalities around Bogotá}

\begin{abstract}
GARCIA, Jhoan M. ${ }^{1}$
FUENTES, Héctor J. ${ }^{2}$

\section{Resumen}

Se expone un análisis de los efectos del COVID 19 a nivel del Departamento de Cundinamarca y más específicamente de los municipios aledaños a Bogotá, usando como información de referencia la base de datos de infectados por el virus del Portal del Estado Colombiano (GOV.CO) y la base de Datos Abiertos https://sig.sispro.gov.co/SituacionCOVID/. Se busca explicar la cantidad de fallecidos en los municipios aledaños a partir de variables como el género, la edad y el tiempo de diagnóstico entre otros. Además, se estiman resultados econométricos en donde se considera tanto las relaciones de carácter social, económico, espacial y territorial que ayudan a comprender de qué forma el distrito capital tiene incidencia en los contagios de los municipios aledaños.

Palabras clave: COVID-19, Cundinamarca, fallecidos, incidencia social
\end{abstract}

\begin{abstract}
An analysis of the effects of COVID 19 at the Department of Cundinamarca and, more specifically, of the municipalities surrounding Bogotá is presented, using reference information the database of infected by the virus of the Colombian State Portal (GOV.CO) and the Open Database https://sig.sispro.gov.co/SituacionCOVID/. It seeks to explain the number of deaths in the neighboring municipalities based on variables such as gender, age, and time of diagnosis, among others. Besides, economic results are estimated where both social, economic, spatial, and territorial relationships are considered to help understand how the capital district has an impact on the contagions of the neighboring municipalities.
\end{abstract}

Key words: COVID-19, Cundinamarca, deceased, social incidence

\section{Introducción}

Cundinamarca es un departamento que cuenta con muchos municipios que rodean las fronteras de Bogotá a excepción de la frontera sureste. Es por ello que la capital y sus alrededores funcionan como todo un sistema interconectado y articulado que afectan de manera directa e indirecta a la población y al espacio así los entornos a la ciudad capital cuenten con una dinámica socio-económica menor. Actualmente, Bogotá ha sido una de las ciudades más afectadas con la pandemia del COVID 19 a pesar de todas las medidas de seguridad que la administración local ha implementado en asocio con la administración departamental de Cundinamarca. Se destaca entre muchas otras, el aislamiento obligatorio y sus consecuencias sobre la ciudad, de la misma manera

\footnotetext{
${ }^{1}$ Ingeniería Catastral y Geodesia. Bogotá. Universidad Distrital Francisco José de Cladas. jmgarciaf@correo.udistrital.edu.co

${ }^{2}$ Docente Titular. Bogotá. Universidad Distrital Francisco José de Caldas. hjfuentes।@udistrital.edu.co
} 
que sus correlaciones espaciales en términos del impacto económico y social para los municipios circunvecinos a la capital (Tono, A. M. O., García, M., Moncayo, C. J., Wills, C., \& Mahecha, Á. M. C., 2020).

De acuerdo con lo anterior, es pertinente analizar de qué forma el sistema dinámico en el que se encuentra Bogotá, afecta el número de contagios por COVID-19 a los municipios aledaños, en especial la cifra de fallecidos y sus probabilidades asociadas, las cuales se calculan a través de un análisis econométrico y que permiten extraer algunas conclusiones para entender a fondo el análisis y el efecto de la pandemia en los alrededores de la ciudad capital.

\section{Marco referencial}

\subsection{Incidencia sanitaria}

Bogotá al ser la ciudad que presentó el primer caso de COVID-19 alertó inmediatamente a los gobiernos distritales y departamentales para adoptar medidas que permitieran la mitigación del virus buscando salvaguardar la vida de la población. Para el mes de septiembre de 2020, Cundinamarca se ubicaba en el sexto lugar del país en casos confirmados, además tenía 73 municipios con registros de contagios de un total de 116 municipios, de los cuales 43 de ellos aún eran considerados municipios no COVID-19 por no haber detectado casos positivos. Según el Ministerio de Salud de Colombia se ha calculado una tasa de contagio de 1.8 acompañada una tasa de letalidad de $2.8 \%$ (Instituto Nacional de Salud, (2020).

Entendiendo la cercanía de los municipios del departamento con el centro de la pandemia en el país, la gobernación de Cundinamarca, ha estado realizando trabajos desde que empezó el aumento de los casos en Bogotá y ha limitando la movilidad de pasajeros y habitantes que salen y entran al departamento, sin descuidar la necesidad de expansión en el número de camas de Unidad de Cuidados Intensivos (UCI), pasando de tener 179 camas a comienzos de la llegada del virus a cerca de 700 camas instaladas en los diferentes municipios de del departamento. (Cundinamarca, COVID-19 - Región Central. (2020).

Se debe destacar la gran cantidad invertida en recursos para la red hospitalaria de Cundinamarca y para el laboratorio de salud pública que suman alrededor de 29.000 millones de pesos). Estos recursos permitieron que el departamento contara con un sistema de salud capaz de responder al aumento de los casos en cuanto al manejo y cuidado de pacientes confirmados con el virus. Es por ello, que la gobernación presentó un detallado balance frente al plan estratégico de expansión de nuevas camas de $\mathrm{UCl}$, que no solo permiten garantizar la atención a la salud de los cundinamarqueses, sino que brindó el 30\% de su capacidad, especialmente en los municipios de Girardot, Soacha, y Chía, para la atención de pacientes provenientes de Bogotá, en momentos en los que la capital casi llegaba al tope en el uso de atención hospitalaria de cuidados intensivos. (Cundinamarca, COVID-19 - Región Central, 2020).

El Instituto Nacional de Salud está en constante actualización de la información y estadísticas descriptivas en los casos confirmados en Colombia, presenta un panorama general del país con cifras de casos confirmados, casos activos, recuperados y fallecidos y hace un enfoque en los fallecidos, dando una breve explicación de como se interpreta el reporte de los fallecidos y qué aspectos se tienen en cuenta para dicha interpretación, consistente en que los reportes de los fallecidos a diario, están divididos en dos grupos, los que han fallecido en días anteriores que no alcanzan a ser reportados el mismo día y los que son reportados el mismo día del anuncio. Este reporte depende de procesos que deben ser rectificados como se indica a continuación:

- "El reporte de las muertes requiere una identificación de la causa para que pueda ser emitido el registro civil de defunción." (Instituto Nacional De Salud, 2020) 
- "El proceso de identificación de la causa, depende del juicio médico la mayoría de las veces, pero en otras se requieren examen de laboratorio, análisis epidemiológicos (tipo autopsia verbal) e incluso autopsia o análisis patológico." (Instituto Nacional De Salud, 2020)

Para el caso de la información departamental, esta se divide en estadísticas y gráficas de contagios por departamento, distribución por sexo, distribución de edad, y un histórico de casos presentado por medio de salidas gráficas de contagios contra el tiempo que ha transcurrido desde el primer caso, comparando los contagios con los recuperados y los fallecidos, la cual se muestra a continuación.

Figura 1

Históricos de casos para el departamento de Cundinamarca

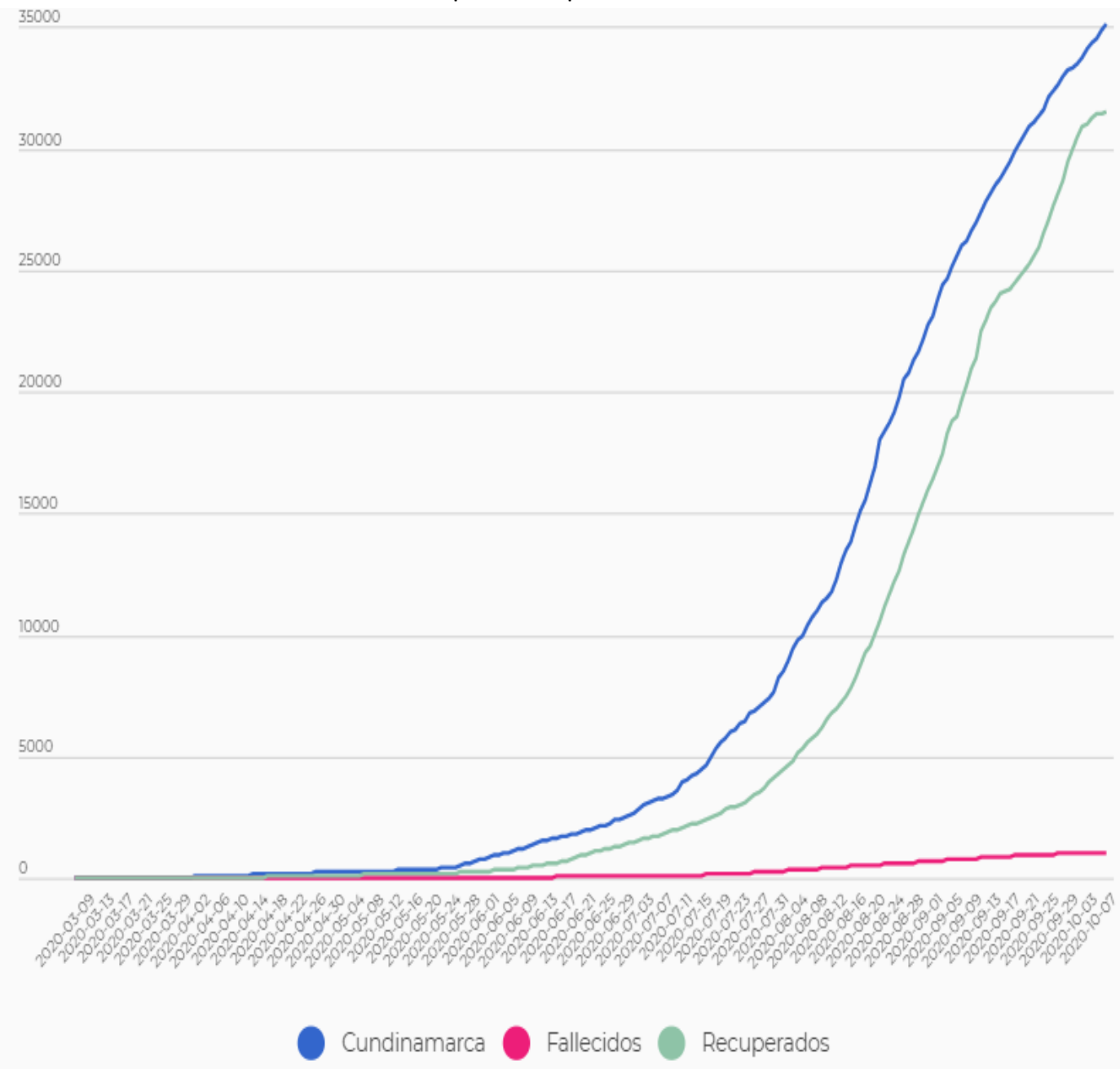

Fuente: Instituto Nacional de Salud https://www.ins.gov.co/Noticias/Paginas/coronavirus-departamento.aspx

En la figura 1 claramente se ve como los contagios y los recuperados siguen una curva muy parecida, pero en diferentes magnitudes. Esto se debe a que no todos los contagiados se recuperan y para ello la curva de fallecidos es muy útil ya que se identifican que de todos los contagios son pocos los que fallecen. Según el Instituto Nacional de Salud para 6 de octubre del 2020 se contaba con 35.087 casos de COVID.19 en el departamento de Cundinamarca, de los cuales 31.476 (89.71\%) son pacientes recuperados, 1.721 (4.9\%) se encuentran en casa, 725 (2.07\%) se encuentran en el hospital, 75 (0.21\%) esta en Unidades de Cuidados Intensivos, 1.043 ( 2.97\%) han fallecidos y $47(0.13 \%)$ han sido muertes ajenas al COVID-19 tal y como se muestra en la figura 2 y 3. 
Figura 2

Distribución por atención por

departamento de Cundinamarca

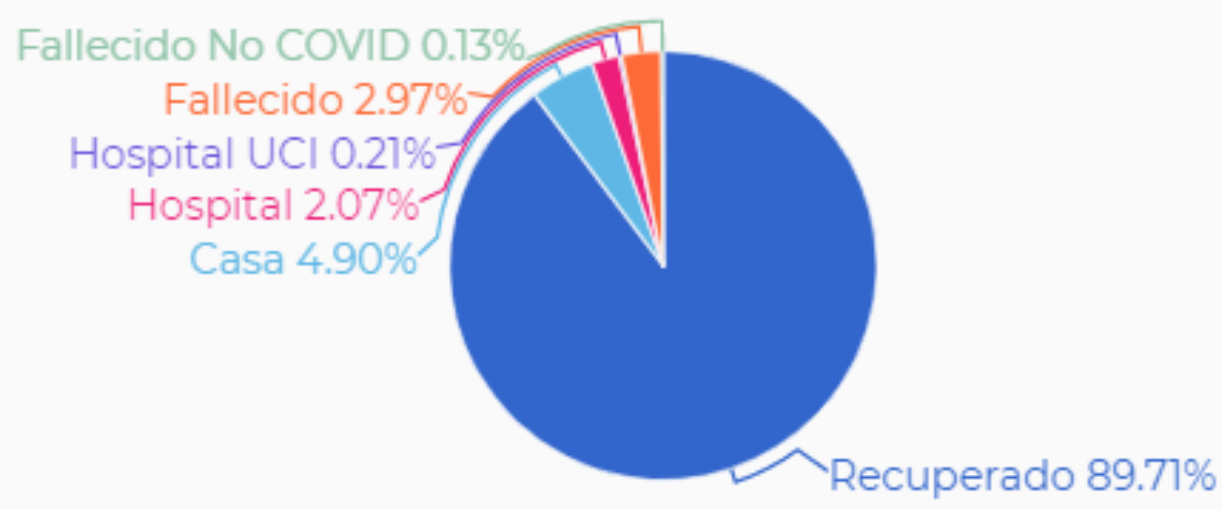

Fuente: Instituto Nacional de Salud https://www.ins.gov.co/Noticias/Paginas/coronavirus-departamento.aspx

Figura 3

Distribución por sexo por departamento de Cundinamarca

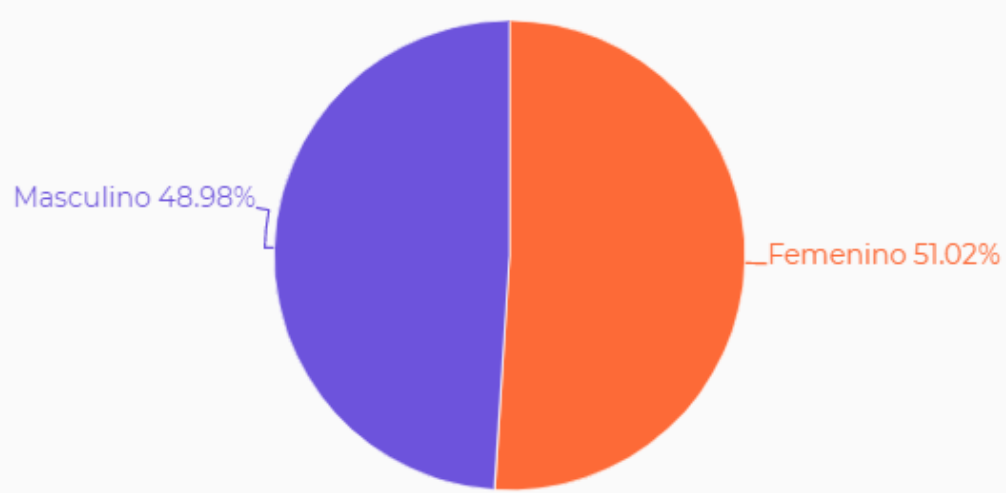

Fuente: Instituto Nacional de Salud https://www.ins.gov.co/Noticias/Paginas/coronavirus-departamento.aspx

Por su parte, en la figura 3 se evidencian los porcentajes de los contagios discriminados por género, siendo el femenino el que más ha contraído contagios con un $2.04 \%$ más que el género masculino.

Al observar los contagios en los municipios aledaños a Bogotá en color rojo, se encuentran los municipios que tienen más de 1.020 contagios y que han sido los de mayor afectación pues coinciden con la cercanía a la capital, foco del mayor brote por COVID en el país. 
Figura 4

Mapa de contagios

en Cundinamarca

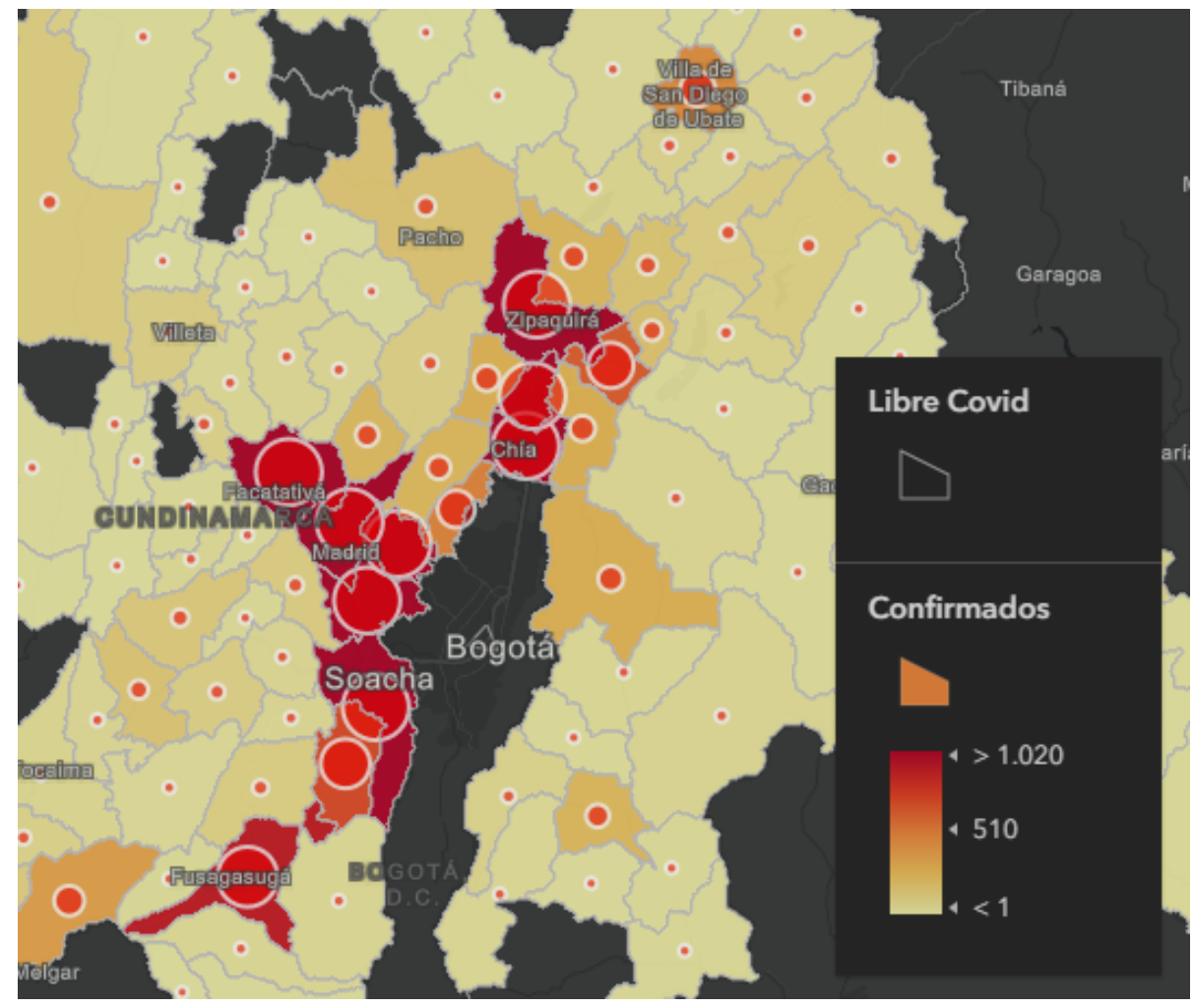

Fuente: Instituto Nacional de Salud

http://saga.cundinamarca.gov.co/apps/COVID/, 2020

\subsection{Incidencia económica}

En el mundo, una de los efectos más impactantes en tiempos de pandemia ha sido la caída de la economía, la cual ha sido una consecuencia lógica del cierre del comercio y del aislamiento decretado por las autoridades con la intensión de disminuir la velocidad a la que se propaga el virus. Los años anteriores a la llegada del virus han sido de relativo crecimiento y según la Cámara de Comercio de Bogotá, en balance económico de la región Bogotá-Cundinamarca para el 2019, fue muy positivo dado que hubo cierta estabilidad macroeconómica, con una inflación del 3.8\% y una inversión extranjera del 25,6\%. Los sectores de mayor aporte correspondieron a las actividades financieras y de seguros $(5,7 \%)$, el comercio al por mayor y por menor $(4,9 \%)$, la administración pública y defensa, educación y salud (4,9\%). A excepción de la construcción todos los sectores presentaron variaciones positivas (De Bogotá, C.D.C. 2020).

Para la región andina de Colombia que incluye tanto al departamento de Cundinamarca como a Bogotá, este territorio se constituye como una de los más atractivos para una buena ubicación empresarial y la realización de diferentes negocios, por aspectos como la diversidad y el dinamismo de sus actividades productivas, además de que dicho territorio está posicionado entre las regiones con mayor producción en América Latina. Para el año 2019 Cundinamarca y Bogotá contaban 504.333 empresas, cifra que representa el 31\% del total de las empresas registradas en Colombia, y que constituyen una demanda de 11 millones de personas entre la capital y el departamento. (De Bogotá, C.D.C. 2020). 
En la siguiente tabla se muestran cifras de empresas matriculadas y renovadas a la jurisdicción de la Cámara de comercio en 2019, en donde se indica que Bogotá cuenta con el $87 \%$ de las 504.333 empresas y Cundinamarca con el $13 \%$ y ubicadas en 59 municipios. Se destacan, Soacha con 3\%, Fusagasugá con 1.4\%, Zipaquirá con 1.2\% y Cajicá con 1\%. La tendencia de inversiones en el sector empresarial se comporta de una manera similar, es decir, Bogotá se lleva más de la mitad del porcentaje de todas las inversiones mientras el resto de municipios cuentan con menor nivel de inversión. En ese orden de ideas, la capital cuenta con el $97 \%$ del total de las inversiones en la región Bogotá-Cundinamarca, mientras los municipios tan solo cuentan con el $3 \%$ sobrante. (De Bogotá, C.D.C. 2020).

\section{Figura5}

Ranking de empresas matriculadas y renovadas jurisdicción de la CCB, 2019.

\begin{tabular}{|c|c|c|c|c|c|c|c|}
\hline $\mathbf{N}^{\circ}$ & Municipio & Microempresa & Pequeña & Mediana & Grande & Total & \%Part \\
\hline 1 & Bogotá D.C. & 387471 & 39040 & 10898 & 3666 & 441075 & $87.5 \%$ \\
\hline 2 & Soacha & 14037 & 237 & 50 & 21 & 14345 & $2.8 \%$ \\
\hline 3 & Chía & 6834 & 617 & 145 & 61 & 7657 & $1.5 \%$ \\
\hline 4 & Fusagasugá & 6930 & 152 & 35 & 3 & 7120 & $1.4 \%$ \\
\hline 5 & Zipaquirá & 5979 & 255 & 47 & 6 & 6287 & $1.2 \%$ \\
\hline 6 & Cajicá & 4050 & 257 & 64 & 21 & 4392 & $0.9 \%$ \\
\hline 7 & Cota & 1827 & 546 & 317 & 111 & 2801 & $0.6 \%$ \\
\hline 8 & Ubaté & 1942 & 116 & 19 & 1 & 2078 & $0.4 \%$ \\
\hline 9 & Tocancipá & 1602 & 118 & 75 & 51 & 1846 & $0.4 \%$ \\
\hline 10 & Sopó & 1163 & 65 & 15 & 9 & 1252 & $0.2 \%$ \\
\hline
\end{tabular}

Fuente: Cámara de Comercio de Bogotá (2019a). Base del Registro Mercantil de la CCB, 2019.

Cálculos: Dirección de Gestión y Transformación de Conocimiento de la CCB

Figura 6

Tamaño de las empresas registradas Bogotá y 59 municipios de Cundinamarca, 2019

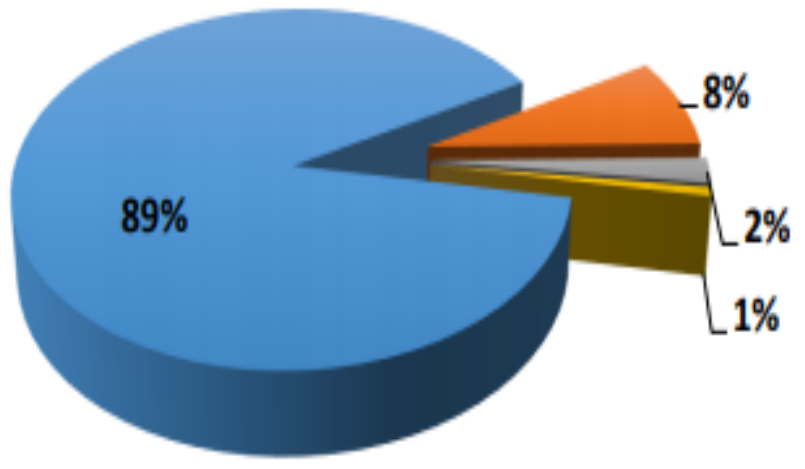

MICROEMPRESAS I PEQUENALAS

Fuente: Cámara de Comercio de Bogotá (2019a). Base del Registro Mercantil de la CCB, 2019.

Cálculos: Dirección de Gestión y Transformación de Conocimiento de la CCB. 
En la región Bogotá-Cundinamarca predominan las micro y pequeñas empresas. Si se tiene en cuenta que el año 2019 desde el punto de vista económico fue importante para el crecimiento de las actividades económicas en la ciudad y sus alrededores, es posible imaginar el impacto que los cerca de 7 meses de confinamiento estricto y de disminución de la demanda agregada de bienes y servicios, han dejado sobre el tejido empresarial de la región.

A través de una encuesta realizada por la Asociación Colombiana de las Micros, Pequeñas y Medianas Empresas (ACOPI), se midieron los efectos económicos del coronavirus en las pequeñas empresas de Bogotá y Cundinamarca, con el objetivo de contribuir con información para los entes distritales, departamentales y nacionales quienes tienen la responsabilidad de diseñar las políticas de recuperación de la economía y el empleo y de reactivar el sector empresarial (Hoyos-Estrada, S., \& Sastoque-Gómez, J. D. 2020)

\section{Figura 7}

Tamaño de empresas de la encuesta

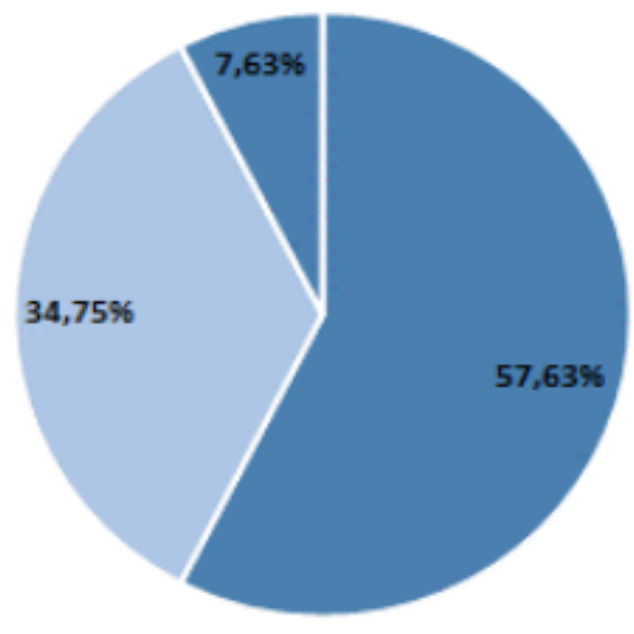

" Pequeña = Micro " Mediana

Fuente: ACOPI (2020). Efectos económicos del coronavirus en las Mipymes de Bogotá y Cundinamarca

Dentro de los hallazgos más importantes de este estudio, se encontró que, del total de empresas encuestadas, el $\mathbf{9 7 . 4 6 \%}$ afirmó estar afrontando tensiones financieras producto de la pandemia. A partir de lo anterior, es evidente que las empresas afrontan una crisis económica, están al borde de caer en la quiebra, trayendo consigo, desempleo, incumplimiento en los pagos mensuales de créditos, y en las obligaciones de pagos de impuestos como el predial, el IVA y renovación en la afiliación a la cámara de comercio. (ACOPI,2020).

Aunque ACOPI adoptó todas las medidas que el Gobierno reglamentó a nivel nacional y local, en la encuesta se manifiesta que los niveles de desempleo serán elevados a corto plazo, requiriéndose de alivios y ayudas en cuanto a las nóminas y a las obligaciones de este tipo como lo son las primas a los trabajadores, ola disminución en los costos parafiscales, la seguridad social, los créditos en entidades bancarias, los pagos de arrendamientos, los servicios públicos, así como la reducción en los precios de alimentos y materias primas. (ACOPI,2020).

En términos del departamento, se han venido diseñando estrategias como líneas de crédito que buscan reactivar el comercio en la región y contribuir a la aceleración de la economía del país. De los 26 municipios del departamento de Cundinamarca con tasas más altas de contagio por COVID-19, las ayudas se han encaminado a reactivar la actividad en los municipios más afectados como lo son: Agua de Dios, Bojacá, Cajicá, Cáqueza, Cota, 
El Rosal, Facatativá, Funza, Fusagasugá, Gachancipá, Girardot, Medina, Mosquera, Nemocón, Nilo, Pacho, Paratebueno, Puerto Salgar, Ricaurte, Silvania, Soacha, Tabio, Tocancipá, Ubalá, Vianí y Zipaquirá. (Region Central RAPE, 2020).

\subsection{Incidencia social}

En la región Bogotá-Cundinamarca habitan aproximadamente 11 millones de personas, alrededor del 21\% del total de la población colombiana. Según los resultados del Censo Nacional de Población y Vivienda publicado por el DANE en el año 2019, la región Bogotá-Cundinamarca es una de las mas pobladas en América latina, situándose en el quinto puesto de las principales áreas metropolitanas.

Una de las características más importantes que intervienen en la región es la distribución de edades. Para la capital, el 24 \% de la población son jóvenes entre 15 y 24 años, que se encuentran en edad de formación escolar y profesional, el $49 \%$ son personas entre 25 a 59 años, edades que generalmente se vinculan al sector productivo o empresarial, es decir, edades laborales. Además, la distribución de la población por género, indica para el año 2018, las mujeres con un 52\%, superaron por un $4 \%$ a la población masculina. Para la región de Cundinamarca, se presentan cifras similares, contando con un $28 \%$ de jóvenes en edad de formación y $45 \%$ de personas en edades laborales y con un $50.2 \%$ de población femenina, que al igual que la capital, supera a la población masculina, en este caso tan solo por $0.4 \%$ tal y como se muestra en la figura 8 . (Cámara de Comercio de Bogotá,2020, p. 96).

Figura 8

Distribución de la población de Cundinamarca según rangos de edad, 2018

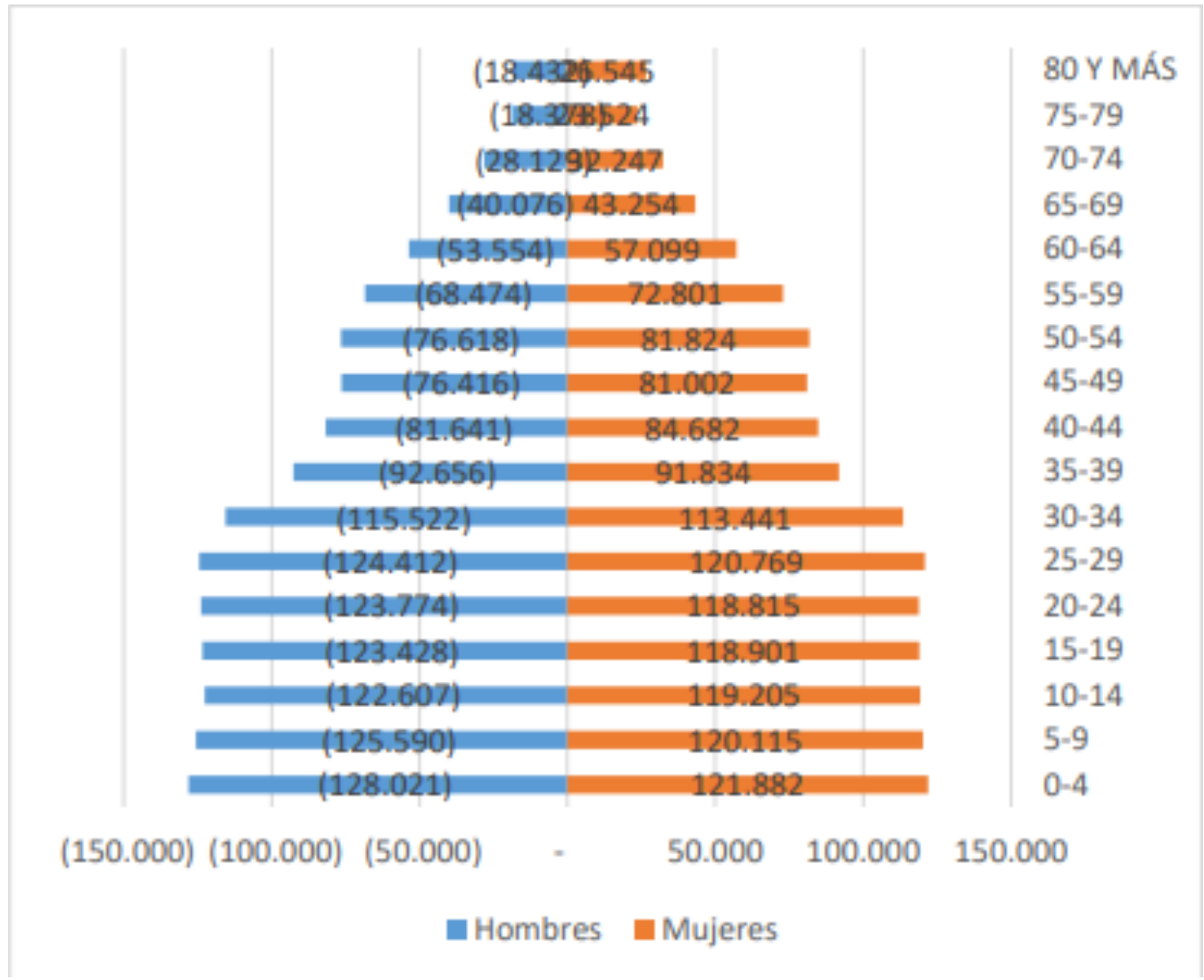

Fuente: DANE - Proyecciones de población 2005-2020 nacional, departamental y municipal por sexo, grupos quinquenales de edad. Elaborado por Dirección de Gestión y Transformación de Conocimiento de la CCB 
Se debe tener en cuenta que en los últimos años en la región Bogotá-Cundinamarca, la pobreza monetaria, es decir la pobreza que mide aquellas personas que integran hogares cuyos gastos per cápita están por debajo del costo de la canasta básica de alimentos, ha generado impacto negativo a la dinámica del sector económico y el social. A continuación, se puede observar en la figura 9 como la pobreza monetaria en Cundinamarca es mayor a la registrada para la ciudad capital, aunque es menor a la del nivel nacional y aunque venía en continuo descenso a partir del 2017, regresó a una tendencia alcista a pesar de tener cerca a la mayor concentración de la industria empresarial y productiva del país (Ramírez, J. M., Bedoya, J. G., \& Díaz, Y., 2016)

\section{Figura 9}

Comportamiento de la pobreza monetaria 2002 - 2018

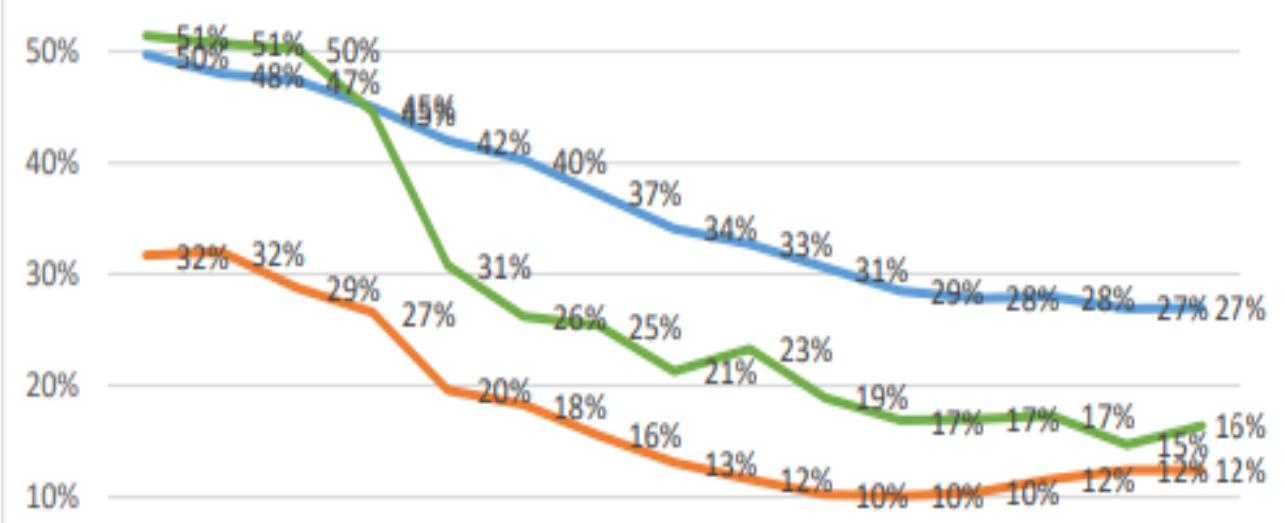

200220032004200520082009201020112012201320142015201620172018

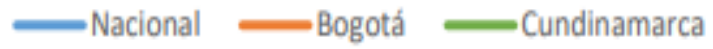

Fuente: DANE - Encuesta Continua de Hogares (2002-2005) y Gran Encuesta Integrada de Hogares (2008-2018). Elaborado por Dirección de Gestión y Transformación de Conocimiento de la CCB

\section{Metodología}

Con el propósito de medir el efecto que la pandemia ha tenido sobre la población y relacionar una aproximación de sus efectos sobre la población en términos de los fallecidos y sus determinantes económicos y sociales, se dispuso de los datos de los casos positivos por COVID-19 en Colombia a través del portal de datos abiertos proporcionados por el Estado colombiano por intermedio del Ministerio de Tecnologías de la Información y las comunicaciones (MinTIC). La base de datos encontrada y denominada casos positivos de COVID-19 en Colombia, está en constante actualización, aunque se decidió tomar los casos representados y manejados hasta el 5 de octubre del 2020.

Esta base de datos cuenta con 22 variables y abarca información como, fechas de reporte, fecha contagio, fecha inicio de síntomas, fecha reporte a las entidades publicas encargadas, género, edad, departamento, municipio, estado del paciente, recuperados y fallecidos entre otras.

Debido a que el análisis está enfocado en la región de Bogotá y sus municipios aledaños, se realizó un filtro por departamento y uno por municipio, escogiendo 20 municipios que cumplen con la característica de estar más cerca en términos de distancia a Bogotá. Estos municipios fueron: Cajicá, Chía, Chipaque, Choachí, Cota, Facatativá, Fómeque, Funza, Fusagasugá, Girardot, La Calera, Madrid, Mosquera, Pasca, Soacha, Sopó, Tenjo, Tocancipá, Ubaque y Zipaquirá, destacando que el más cercano es Soacha a 19 Km y el más lejano Girardot a 140 $\mathrm{km}$. 
Luego de tener la base de datos con las variables a usar en el modelo, se realizó un análisis econométrico tipo modelo probabilístico con el fin de indagar sobre los determinantes del fallecimiento de los contagiados por el nuevo coronavirus y evidenciar los efectos que constituye para estos territorios, su cercanía con la capital, que aunque se ve beneficiada por tener un importante mercado para la venta de la producción del departamento, también se ve afectada por estar tan cerca del principal foco de contagio del país.

Para este propósito, se ha buscado una herramienta de carácter cuantitativo para medir una respuesta sobre una variable cualitativa que permite estimar las probabilidades de ocurrencia de un evento a partir del comportamiento de variables explicativas. Los métodos clásicos por excelencia corresponden a los modelos probit y logit, que son alternativas más eficientes para la estimación de probabilidades, que los modelos lineales de probabilidad. Dentro de las principales diferencias y bondades de los modelos probabilísticos logit y probit frente a los modelos lineales están las condiciones para estimar probabilidades entre 0 y 1 , así como considerar que los cambios en probabilidad no son lineales y las tasas de cambio son cada vez más lentas cerca del cero y cerca del uno (Gujarati, 2009)

En términos del modelo probit se considera que existe una variable no observable que puede inferir en el comportamiento de la variable explicativa, la cual es determinada por una o varias variables independientes, de forma que se tenga una proporción directa entre la variable latente y la regresada; este modelo sigue una distribución Normal (Wooldrigde, 2001), por lo cual si:

$$
Z_{i}=\boldsymbol{\beta}_{\mathbf{0}}+\boldsymbol{\beta}_{\mathbf{1}} \boldsymbol{x}_{\boldsymbol{i}}+\boldsymbol{\mu} \text { Con } x_{i} \text { la variable regresora }
$$

Entonces

$$
P_{i}=P(Y=1 / x)=P\left(Z_{i} \leq \boldsymbol{\beta}_{\mathbf{0}}+\boldsymbol{\beta}_{\mathbf{1}} \boldsymbol{x}_{\boldsymbol{i}}+\boldsymbol{\mu}\right)
$$

Pero como la variable $Z$ en este caso sigue una distribución normal estándar, es decir con media cero $\mu=0$ y varianza $\sigma^{2}=1$, entonces en general la función de distribución acumulada es:

$$
f(z)=\int_{0}^{z_{i}} \frac{1}{\sqrt{2 \pi \sigma^{2}}} e^{-\frac{(z-\rho)^{2}}{2 \sigma^{2}}} d z=\int_{0}^{\beta_{0}+\beta_{1} x_{i}+\mu} \frac{1}{\sqrt{2 \pi}} e^{-\frac{z^{2}}{2}} d z
$$

La estimación de este tipo de modelos se hace a través de máxima verosimilitud cuyo propósito es obtener los valores de los parámetros de manera que la probabilidad de observar las $Y$ sea lo más grande posible. Este procedimiento es similar tanto para los modelos probit como para los modelos logit, distinguiéndose únicamente en la función de densidad acumulada para los primeros, y la función de densidad acumulada logística para los segundos.

Los resultados del modelo econométrico propuesto se corren a través del programa STATA versión 14.0 que permitieron obtener los resultados de probabilidad de fallecer por COVID-19 en función de las variables género que distingue a paciente femenino y masculino, edad del paciente en años, tiempo de diagnóstico en días, y ubicación espacial del paciente en términos del municipio en el que habita.

\section{Resultados y análisis}

La tabla 1 muestra los resultados de los modelos estimados. En esta tabla se encuentran las salidas de un modelo probit y se complementa con los de un modelo logit. Se adjuntan los cambios de probabilidad para los dos modelos, e igualmente las razones de probabilidad (odds ratio) para el caso del logit. Se adjuntan igualmente los errores estándar y se resaltan en asterisco los valores estimados significativos al 10, 5 y $15 \%$ respectivamente. Se destaca de la tabla 1, las cuatros columnas que muestran resultados para la probabilidad de fallecer así: la columna uno y tres muestran las salidas de los modelos logit y probit en las que se destaca el signo positivo o 
negativo que representa un aumento o disminución en la probabilidad para cada variable. A su vez, las columnas 2 y 4 muestran los cambios en probabilidad para el modelo probit y los odds ratio o razones de probabilidad para el caso del modelo logit.

Tabla 1

Salida econométrica de

los modelos Logit y Probit

\begin{tabular}{|c|c|c|c|c|}
\hline & PROBIT & DPROBIT & LOGIT & LOGISTIC \\
\hline Variables & fallecido & fallecido & fallecido & fallecido \\
\hline \multirow[t]{2}{*}{ hombre } & $0.403 * * *$ & $0.00668^{* * *}$ & $0.840 * * *$ & $2.316^{* * *}$ \\
\hline & -0.0388 & -0.000775 & -0.0814 & -0.0814 \\
\hline \multirow[t]{2}{*}{ edad } & $0.0450 * * *$ & $0.000702^{* * *}$ & $0.0939 * * *$ & $1.098^{* * *}$ \\
\hline & -0.00121 & $-4.52 \mathrm{E}-05$ & -0.00246 & -0.00246 \\
\hline \multirow[t]{2}{*}{ Chia } & -0.0355 & -0.000534 & -0.0893 & 0.9145292 \\
\hline & -0.127 & -0.00184 & -0.27 & -0.27 \\
\hline \multirow[t]{2}{*}{ Chipaque } & 0.593 & 0.02 & 1.06 & 2.887651 \\
\hline & -0.383 & -0.0226 & -0.79 & -0.79 \\
\hline \multirow[t]{2}{*}{ Choachi } & $1.090^{* * *}$ & 0.0672 & $1.972^{* *}$ & $7.188^{* *}$ \\
\hline & -0.404 & -0.0556 & -0.858 & -0.858 \\
\hline \multirow[t]{2}{*}{ Cota } & 0.153 & 0.00289 & 0.0679 & 1.070243 \\
\hline & -0.184 & -0.00415 & -0.419 & -0.419 \\
\hline \multirow[t]{2}{*}{ Facatativa } & 0.125 & 0.00224 & 0.205 & 1.227761 \\
\hline & -0.132 & -0.0027 & -0.282 & -0.282 \\
\hline \multirow[t]{2}{*}{ Fómeque } & 0.191 & 0.00381 & 0.448 & 1.565897 \\
\hline & -0.546 & -0.0136 & -1.1 & -1.1 \\
\hline \multirow[t]{2}{*}{ Funza } & $0.317^{* *}$ & $0.00717^{*}$ & $0.619 * *$ & $1.857^{* *}$ \\
\hline & -0.128 & -0.00397 & -0.269 & -0.269 \\
\hline \multirow[t]{2}{*}{ Fusagasuga } & 0.0215 & 0.000345 & 0.013 & 1.013131 \\
\hline & -0.139 & -0.00229 & -0.287 & -0.287 \\
\hline \multirow[t]{2}{*}{ Girardot } & $0.404 * * *$ & $0.0101 * *$ & $0.814^{* * *}$ & $2.257^{* * *}$ \\
\hline & -0.12 & -0.00446 & -0.25 & -0.25 \\
\hline \multirow[t]{2}{*}{ La Calera } & $0.417^{*}$ & 0.0111 & 0.833* & $2.300^{*}$ \\
\hline & -0.213 & -0.00868 & -0.445 & -0.445 \\
\hline \multirow[t]{2}{*}{ Madrid } & $0.310^{* *}$ & $0.00695 *$ & $0.616^{* *}$ & $1.851^{* *}$ \\
\hline & -0.134 & -0.00412 & -0.288 & -0.288 \\
\hline \multirow[t]{2}{*}{ Mosquera } & $0.241^{*}$ & 0.00493 & $0.487^{*}$ & $1.627^{*}$ \\
\hline & -0.124 & -0.00322 & -0.263 & -0.263 \\
\hline \multirow[t]{2}{*}{ Pasca } & 0.262 & 0.00573 & 0.527 & 1.69464 \\
\hline & -0.656 & -0.0193 & -1.194 & -1.194 \\
\hline \multirow[t]{2}{*}{ Soacha } & $0.334^{* * *}$ & $0.00597^{* * *}$ & $0.708^{* * *}$ & $2.030 * * *$ \\
\hline & -0.107 & -0.00223 & -0.229 & -0.229 \\
\hline \multirow[t]{2}{*}{ Sopo } & $0.420^{* *}$ & 0.0112 & $0.845^{* *}$ & $2.328^{* *}$ \\
\hline & -0.202 & -0.00831 & -0.419 & -0.419 \\
\hline \multirow[t]{2}{*}{ Tenjo } & 0.209 & 0.00425 & 0.378 & 1.459258 \\
\hline & -0.252 & -0.00651 & -0.538 & -0.538 \\
\hline \multirow[t]{2}{*}{ Tocancipa } & -0.0629 & -0.000911 & -0.108 & 0.8975 \\
\hline & -0.202 & -0.0027 & -0.439 & -0.439 \\
\hline \multirow[t]{2}{*}{ Ubaque } & $1.276^{* *}$ & 0.0966 & $2.272^{*}$ & 9.700* \\
\hline & -0.599 & -0.106 & -1.352 & -1.352 \\
\hline Zipaquira & 0.15 & 0.00274 & 0.202 & 1.223 \\
\hline
\end{tabular}




\begin{tabular}{|c|c|c|c|c|}
\hline & PROBIT & DPROBIT & LOGIT & LOGISTIC \\
\hline Variables & fallecido & fallecido & fallecido & fallecido \\
\hline & -0.119 & -0.00251 & -0.253 & -0.253 \\
\hline Tiempodiagnóstico & $-0.00728^{*}$ & $-0.000114^{*}$ & $-0.0147^{*}$ & $0.9854^{*}$ \\
\hline & -0.0039 & $-6.14 \mathrm{E}-05$ & -0.00877 & -0.0087 \\
\hline Constant & $-4.662^{* * *}$ & & $-9.370^{* * *}$ & $0 * * *$ \\
\hline & -0.134 & & -0.288 & -0.288 \\
\hline & & & & \\
\hline Observations & 29,111 & 29,111 & 29111 & 29111 \\
\hline
\end{tabular}

Fuente: Elaboración propia

Sobresale de los resultados anteriores, como las variables más relevantes que explican el propósito de este trabajo son el sexo, la edad, el tiempo de diagnóstico, así como los municipios de Ubaque, Sopó, Choachí, Funzá, Girardot, Madrid, Mosquera, Soacha. Dada la cercanía de estos municipios a la ciudad de Bogotá y que sus habitantes tienen una interacción importante con la capital tanto en términos laborales como den términos de comercio, es muy probable que dicha cercanía se un factor crucial de contagio por cercanía. En el caso de ciudades como Girardot y Choachí, que son de destino turístico por tierra y sus desplazamientos son relativamente fáciles para los bogotanos, se muestra cierto patrón de correlación entre los contagios de la capital.

Del mismo modo, la tabla 1 igualmente en la última columna, que las probabilidades de fallecimiento por COVID19 para los municipios aledaños a al capital son en su orden de mayor a menor: Ubaque (a $60 \mathrm{Km}$ de Bogotá), Choachí (a $53.7 \mathrm{Km}$ de Bogotá) , Chipaque ( a 45.7 de Bogotá) , La Calera (a 24.9 km de Bogotá) , Sopó (a 36.9 km de Bogotá), y Girardot ( a $140 \mathrm{Km}$ de Bogotá), Soacha ( a $19.2 \mathrm{Km}$ de Bogotá), Funza ( a $22.6 \mathrm{Km}$ de Bogotá), Madrid ( a $30 \mathrm{Km}$ de Bogotá), Mosquera (a $25.3 \mathrm{~km}$ de Bogotá), que son los municipios con una razón de probabilidades más altas que la de los demás municipios.

Dentro de las mayores probabilidades de fallecimiento por COVID y como se muestra en las razones de probabilidad estimadas, el ser hombre representa una probabilidad de más del doble (2.3) de fallecer que el de una mujer. Para el caso de la edad, se muestra que un año de edad de más en un paciente aumenta la probabilidad de fallecer en un $0.07 \%$.

Figura 10

Gráfica ROC para comparación

de modelos Logit y Probit
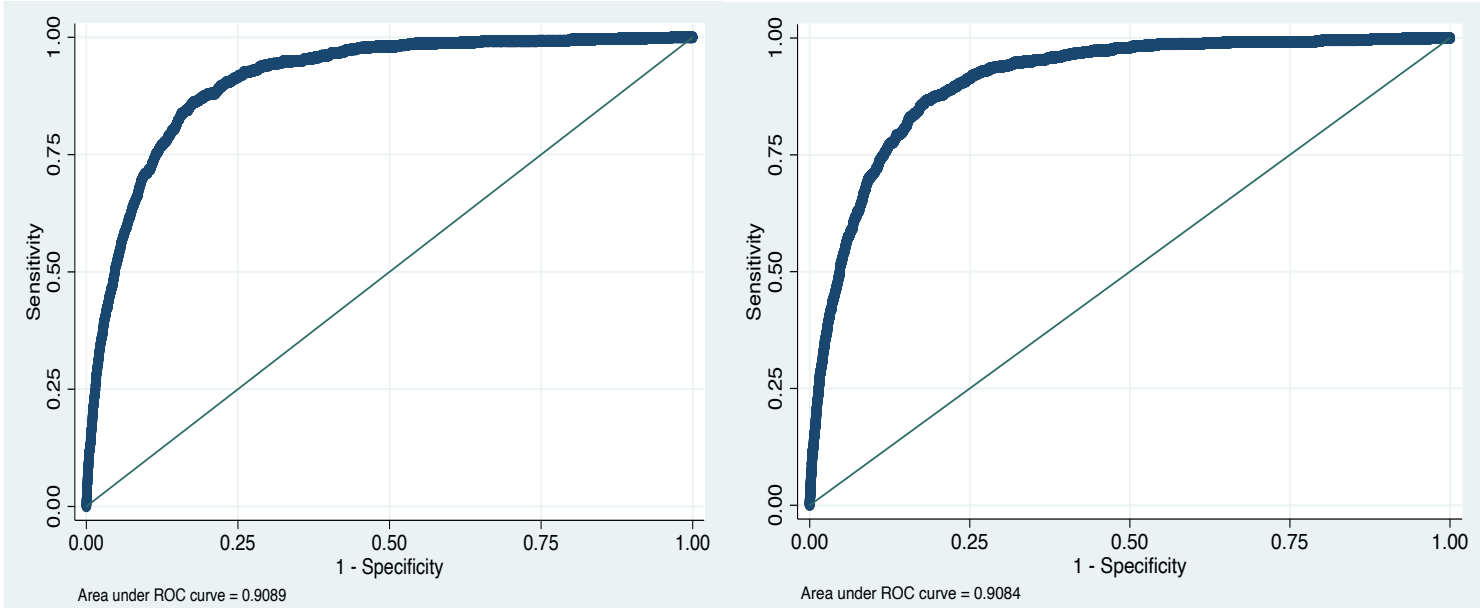

Fuente: Elaboración propia 
De la figura 10 se puede observar la curva de ajuste de los valores arrojados por los modelos frente a los valores observados, encontrandose que la diferencia entre un modelo logit y probit no es relevante y en consecuencia los dos aportan resultados validos con una tasa de pronóstico del 90,89\% y del 90,84\% respectivamente. Lo cual es un indicador de que los valores que los modelos arrojan ajustan de manera significativa a los valoers observados de la muestra.

\section{Conclusiones}

Bogotá es una ciudad que a lo largo de la historia ha tenido un contundente crecimiento tanto en lo territorial como en lo económico que ha generado impactos sociales positivos y negativos. De esto se colige que la capital y sus vecinos actúan como una gran área metropolitana en la que se permea sus condiciones de progreso y así como sus problemáticas sociales, ambientales y sanitarias dado que conforman un solo sistema.

La relación del sistema conformado por la región Bogotá-Cundinamarca y todo lo que se ha venido trabajando en la actualidad referente al COVID-19, ha sido muy importante. La integración de políticas locales y departamentales indican que la región Bogotá-Cundinamarca ha podido coordinar esfuerzos en pro de mitigar los efectos de la pandemia, no obstante, las mayores afectaciones en términos económicos son compartidas por la capital y su entorno, en especial en lo referente a las micro, pequeñas y medianas empresas industriales y comerciales.

Se destaca que la coordinación en políticas de salud ha permitido mantener tasas de fallecimientos en bajos niveles tanto para la capital como para el departamento, y no se pueden desconocer, que muchos de estos decesos corresponden a personas mayores de género masculino y en municipios cercanos a la ciudad capital, es decir, obedecen a una interacción con la capital pero se destaca la mayor edad de los fallecidos, quienes por lo general tienen asociados comorbilidades que aumentan el riesgo de fallecimiento.

Se puede subrayar que, a pesar de ser el quinto país del mundo con más infectados al mes de octubre de 2020 según los reportes dados por el Hospital universitario John Hopkins, la tasa de mortalidad del COVID-19, no ha sido tan marcada como la de los países con menos infecciones y mejores sistemas de salud. En el caso Bogotá y sus vecinos más cercanos las tasas de mortalidad mantienen una estrecha relación que se explica igualmente por su cercanía espacial y su grado de interacción diaria.

\section{Referencias bibliográficas}

Asociación colombiana de las micro, pequeñas y medianas empresas. (2020). Efectos económicos del coronavirus en las mipymes de Bogotá y Cundinamarca. Encuesta. Recuperado de: https://acopibogota.org.co/wp-content/uploads/2020/03/Encuesta-An\%C3\%A1lisis-yPresentaci\%C3\%B3n-de-encuesta-Sobre-Percepci\%C3\%B3n-del-COVID-19.pdf

Cámara de Comercio de Bogotá, (2019a). Base del Registro Mercantil de la CCB, 2019. Cálculos: Dirección de Gestión y Transformación de Conocimiento de la CCB.

Cundinamarca, COVID-19 - Región Central. (2020). Región Central. Recuperado de: https://regioncentralrape.gov.co/cundinamarca-COVID-19/. De Bogotá, C. D. C. (2020). Balance de la economía de la región Bogotá-Cundinamarca 2019.

DANE (2020) - Proyecciones de población 2005-2020 nacional, departamental y municipal por sexo, grupos quinquenales de edad. Elaborado por Dirección de Gestión y Transformación de Conocimiento de la CCB.

DANE (2019). Encuesta Continua de Hogares (2002-2005) y Gran Encuesta Integrada de Hogares (2008-2018). Elaborado por Dirección de Gestión y Transformación de Conocimiento de la CCB. 
Gujarati, D., \& Porter, D. (2010). Econometría (quinta edición). México: McGRAW-HILL/Interamericana Editores, SS DE CV.

Hoyos-Estrada, S., \& Sastoque-Gómez, J. D. (2020). Marketing Digital como oportunidad de digitalización de las PYMES en Colombia en tiempo del COVID-19. Revista científica anfibios, 3(1), 39-46

Instituto Nacional de Salud. (2020). Portal del Estado Colombiano. COVID-19 en Colombia. Recuperado de: https://www.ins.gov.co/Noticias/Paginas/coronavirus-departamento.aspx

Ramírez, J. M., Bedoya, J. G., \& Díaz, Y. (2016). Geografía económica, descentralización y pobreza multidimensional en Colombia.

Tono, A. M. O., García, M., Moncayo, C. J., Wills, C., \& Mahecha, Á. M. C. (2020). CoVID-19: generalidades, comportamiento epidemiológico y medidas adoptadas en medio de la pandemia en Colombia. ACTA DE OTORRINOLARINGOLOGÍA \& CIRUGÍA DE CABEZA Y CUELLO, 4-13.

Wooldridge, J. M. (2010). Econometric analysis of cross section and panel data. MIT press.

Esta obra está bajo una Licencia Creative Commons Attribución-NoCommercial 4.0 International

\section{(cc) EY-NC}

